\title{
Is There Any Effect of Diagnostic Disease Activity Index on Current Myocardial Function in Pediatric Inflammatory Bowel Disease Patients?
}

\author{
(1) Esra Polat ${ }^{1}$, (D) Elif Erolu², (1) Nelgin Gerenli1', (1) Hasret Ayyıldız Civan³ \\ 1University of Health Sciences Turkey, İstanbul Ümraniye Training and Research Hospital, Clinic of Pediatrics, Division of Pediatric Gastroenterology, \\ İstanbul, Turkey \\ 2University of Health Sciences Turkey, İstanbul Ümraniye Training and Research Hospital, Clinic of Pediatrics, Division of Pediatric Cardiology, \\ İstanbul, Turkey \\ 3University of Health Sciences Turkey, İstanbul Bakırköy Dr. Sadi Konuk Training and Research Hospital, Clinic of Pediatrics, Division of Pediatric \\ Gastroenterology, İstanbul, Turkey
}

\begin{abstract}
Aim: Inflammatory bowel disease (IBD): [Crohn's disease (CD) or ulcerative colitis (UC)] is characterised by chronic inflammation with multisystemic effects. Myocardial dysfunction may occur during active disease due to increased inflammation. The aim of our study is to evaluate the effect of disease activity index at diagnosis on myocardial functions in children with IBD in remission.

Materials and Methods: The study consisted of 34 IBD patients and 21 healty children. Patients were divided into two groups according to disease activity at the diagnosis as mild and severe disease activity. Conventional echocardiography and strain echocardiography was performed for all study patients.

Results: In IBD patients with severe disease activity at diagnosis, global longitudinal strain of left ventricle (GLLV), longitudinal strain (AP3) and (AP4) were lower than patients with mild disease activity at the diagnosis ( $p=0.043, p=0.032, p=0.028$, respectively). Disease activity at the diagnosis was inversely correlated to ejection fraction (\%), strain values of GLLV, left ventricle apical chamber (LVAP) 3 , LVAP2 in UC patients. $(p=0.047, p=0.016, p=0.029, p=0.011$, respectively). Mitral $E$, DT was lower in IBD patients than control patients $(p=0.05, p=0.04)$. Strain value of LVAP4 was lower in CD patients, than UC patients ( $p=0.046)$. Strain values of GLLV, LVAP3, LVAP4 was decreased in CD patients than control patients ( $p=0.046, p=0.046, p=0.048$ ). Mitral $E$ and desceleration time were found to be decreased in IBD patients than control patients $(p=0.05, p=0.04$ respectively).
\end{abstract}

Conclusion: Our study revealed that the severity of disease activity at diagnosis has long-term effects on myocardial functions.

Keywords: Inflammation, left ventricular function, strain echocardiography

\section{Introduction}

Inflammatory bowel disease (IBD): [Crohn's disease (CD) or ulcerative colitis (UC)] is the result of an immune response in individuals with genetic predisposition. It is thought that luminal antigens cause systemic immunoreaction due to increased intestinal permeability. Triggered systemic inflammation results in fibrosis and dysfunction in the extraintestinal organs $(1,2)$. Inflammation has effects on

Address for Correspondence

Esra Polat, University of Health Sciences Turkey, İstanbul Ümraniye Training and Research Hospital, Clinic of Pediatrics, Division of Pediatric Gastroenterology, İstanbul, Turkey

Phone: +90 5055500170 E-mail: esrkcdr@gmail.com ORCID: orcid.org/0000-0002-0185-0344

Received: 27.04.2021 Accepted: 06.07.2021

- Copyright 2022 by Ege University Faculty of Medicine, Department of Pediatrics and Ege Children's Foundation

The Journal of Pediatric Research, published by Galenos Publishing House. 
cardiac structures either in an occult or overt manner. In acute exacerbation periods, pericarditis, myocarditis, arteritis, valvular regurgitation and tromboembolic events can be seen (3-5). Subtle myocardial involvement was shown in a few studies due to ongoing chronic low grade inflammation (6-8). We studied myocardial functions with conventional echocardiography and strain echocardiography, which gives more reliable information about global and segmental myocardial function. We investigated the effect of the severity of disease activity at the time of diagnosis on current myocardial functions by strain echocardiography. Patients in a remission period were chosen to exclude the effects of any increased inflammation of the active disease on myocardium.

\section{Materials and Methods}

Of the 42 IBD patients followed up in two different paediatric gastroenterology centres, 34 patients in remission were included in this study. Additionally, twentyone healthy children were evaluated as a control group. The control group comprised patients referred to a paediatric gastroenterology clinic with non-specific abdominal pain with no systemic or cardiovascular disease. The diagnosis of 16 patients in the study group was CD and 18 of them were UC. The inclusion criteria of the study group was being in a remission period. IBD diagnosis was made based on clinical symptoms, radiological, endoscopic, and histopathological features according to the Montreal Classification and revised Porto Criteria $(9,10)$. Paediatric CD activity index and paediatric UC activity index were recorded from the patients' medical records $(11,12)$. The actual height, weight and blood pressure of all the patients were recorded. Body mass index (BMI) was obtained by means of the formula weight $(\mathrm{kg})$ / height $^{2}(\mathrm{~m})$. Patients groups were established according to disease activity at the time of diagnosis as either mild or severe disease activity. There were no patients with moderate disease activity at the time of diagnosis. Written consent for the study was obtained from the parents of all the patients and this study was approved by the local ethics committee of the University of Health Sciences Turkey, Kanuni Sultan Süleyman Training and Research Hospital (approval no: 06, date: 29.06.2018).

\section{Echocardiographic Protocols and Definitions}

Echocardiographic evaluation was performed using a Philips Affiniti 50C (Release 2.0.2 3000 Minuteman Road Andover, MA 01810 USA) echocardiography machine and 8-4 Mhz transducer in all subjects. Left ventricular diameters and systolic functions were assessed by M-mode echocardiography at a parasternal long axis view. Pulsed wave Doppler was performed to determine the mitral inflow parameters, namely, mitral $E$ velocity, mitral $A$ velocity, interventricular contraction time and deceleration time (DT).

For strain analysis, standard 2D grayscale images of LV were performed. Apical 4-chamber, 3-chamber and 2-chamber (AP4, AP3 AP2, respectively) views were obtained for the longitudinal strain of LV as previously described (13). Circumferential strain was obtained by parasternal shortaxis views at the mitral valve plane, papillary muscle plane and apical plane (SAXB, SAXM, SAXA, respectively).

Synchronized with continuous electrocardiography, five consecutive cardiac cycles were recorded with frame rate of 60 and 90 frames per second. At end-systole, seven segments for longitudinal strain and six segments for circumferential strain were assessed by tracing the endocardial border line manually. Global longitudinal strain was obtained from the apical four-three-two chamber views and circumferential strain was obtained from the basal, mid-cavity, apical short axis views using speckle-tracking echocardiography software package (QLAB advanced ultrasound quantification soft release 10.4).

\section{Statistical Analysis}

Twenty-two software package (IBM Corp., Armonk, NY, USA) was used for the analysis of the data. The KolmogorovSmirnov test was used to determine the distribution of normality. Data is expressed as mean \pm standard deviation for variables; number and percentages (\%) are given for categorical variables, Mann-Whitney $U$ and Student's t-test were used for the comparison of groups, whichever being more appropriate. For the analysis of correlations, the Spearman rank correlation coefficient was used. Statistical significance was considered at a p-value $<0.05$.

\section{Results}

This study consisted of 34 IBD patients (median: 11 years, range: $5-17$ years, $10.9 \pm 3.9$ years of age; 16 CD and 18 UC) and 21 healthy children (median: 12.5 years, range: 5-18 years, $11.6 \pm 4$ years of age) as a control group $(p=0.15)$. All patients in the IBD and control groups and in the subgroups of severe or mild disease activity were comparable in terms of age, sex, height, weight and BMI. Their demographic data is shown in Table I.

Eleven patients were undergoing mesalamine therapy, 23 patients were receiving mesalamine and azathiopurine therapy, 3 patients were being treated with mesalamine 
and infliximab and 1 patient was receiving treatment with mesalamine, azathiopurine and infliximab. The duration of the disease was $29.4 \pm 23.4$ months (median: 28 months; range: 3-96 months), the duration of treatment was 23.8 \pm 19.8 months (median: 23 months; range: 1-84 months), the activity index at the time of diagnosis was $51.3 \pm 12.8$ (median: 50; range: $35-85$ ), the activity index at the time of test was 7.1 \pm 8.4 (median: 5; range: 0-30). There were 3 patients who had perianal disease, 4 patients who had extra intestinal disease, and one patient who had perianal and extra intestinal disease.

Four $C D$ and 12 UC patients had mild activity index and $12 \mathrm{CD}$ and $6 \mathrm{UC}$ patients had severe activity index at the time of diagnosis.

There were no statistically significant differences between the groups and subgroups in terms of left ventricular diameters, ejection fraction (EF\%), and shortening fraction (SF\%). Mitral inflow parameters revealed decreased mitral E and DT in IBD patients in comparison to the control patients $(p=0.05, p=0.04)$ (Table II). DT was lower in IBD patients with severe disease activity in comparison to the control group and those IBD patients with mild disease activity $(p=0.006, p=0.05)$ (Table III). Mitral $E$ was lower in CD patients than in the control patients $(p=0.038)$ and DT was lower in UC patients than in the control patients $(p=0.02)$. Other conventional echocardiographic parameters were not statistically significantly different between the UC, CD and control group patients (Table IV).

\section{Strain Echocardiography}

There was no statistical difference between the IBD and control patients in terms of the strain parameters of the left ventricle (Table II).
The strain value of LVAP4 was lower in those patients with CD $(-17.1 \pm 3.3)$ than in patients with UC $(-19.1 \pm 2.3)$ $(p=0.046)$ (Table IV). The GLLV, LVAP4, and LVAP3 strain values were lower in the $C D$ group than in the control patients ( $p=0.046, p=0.048, p=0.046$ respectively).

In patients with severe disease activity at diagnosis, the strain values of GLLV $(-17.1 \pm 2.9$ vs $-18.7 \pm 1.79)$, LVAP3 $(-16.4 \pm 3.24$ vs $-19 \pm 2.8)$ and LVAP4 $(-17.8 \pm 3.5$ vs $-19 \pm 2.5)$ were lower than in those patients with mild disease activity at the time of diagnosis $(p=0.043, p=0.032$, $p=0.028$, respectively). In addition, the strain values of GLLV $(-17.1 \pm 2.9$, vs $-18 \pm 2.04)$ LVAP3 $(-16.4 \pm 3.24$ vs $-17.7 \pm 2.7)$, and LVAP4 (-17.8 \pm 3.5 vs $-18.5 \pm 2.1)$ were lower in those IBD patients with severe disease activity than in the control patients $(p=0.044, p=0.018, p=0.028$, respectively) (Table III).

Disease activity at the time of diagnosis was inversely correlated to $E F(\%)(p=0.047 r=-0.50)$ and the strain values of GLLV ( $p=0.016, r=-0.57)$, LVAP3 $(p=0.029, r=-0.53)$, and LVAP2 $(p=0.011, r=-0.53)$ in UC patients.

\section{Discussion}

IBD is a chronic inflammatory immune mediated disease with acute exacerbation and remission periods. Chronic inflammation exists systemically, extending beyond the gut in addition to local inflammation. The status of chronic inflammation is the main cause of extra intestinal manifestations. Cardiac involvement is often in the form of pericarditis and myocarditis during acute exacerbation periods (14-16). Endocarditis, atrioventricular block and arrhythmias are the other forms of cardiac involvement in $\operatorname{IBD}(17,18)$. Subtle myocardial involvement in IBD patients was shown in a few studies via strain echocardiography

Table I. Demographic and echocardiographic features of study patients

\begin{tabular}{|c|c|c|c|c|c|c|}
\hline & \multicolumn{4}{|c|}{ Demographic features of study patients } & \multirow[b]{2}{*}{$\mathbf{p}^{*}$} & \multirow[b]{2}{*}{$\mathbf{p}^{\dagger}$} \\
\hline & Mild disease activity & Severe disease activity & Control & p-value & & \\
\hline Gender (M/F) & $8 / 7$ & $10 / 9$ & $10 / 11$ & 0.57 & 0.35 & 0.47 \\
\hline Age (years) & $9.9 \pm 2.3$ & $11.9 \pm 2.8$ & $11.6 \pm 4.02$ & 0.15 & 0.23 & 0.68 \\
\hline Weight (kg) & $38 \pm 10$ & $38.8 \pm 9.5$ & $39.4 \pm 17$ & 0.25 & 0.75 & 0.46 \\
\hline Height $(\mathrm{cm})$ & $139 \pm 24$ & $141.3 \pm 19.4$ & $145.3 \pm 22.8$ & 0.54 & 0.64 & 0.37 \\
\hline BMI & $15.9 \pm 2.4$ & $16.8 \pm 1.7$ & $17.6 \pm 2.8$ & 0.10 & 0.35 & 0.78 \\
\hline $\mathrm{SBP}(\mathrm{mmHg})$ & $98 \pm 7.8$ & $105 \pm 6.7$ & $101 \pm 9.7$ & 0.19 & 0.44 & 0.62 \\
\hline $\mathrm{DBP}(\mathrm{mmHg})$ & $59 \pm 8.9$ & $62 \pm 9.2$ & $60.4 \pm 10.4$ & 0.09 & 0.12 & 0.38 \\
\hline Pulse Pressure & $41 \pm 6.7$ & $40.9 \pm 5.8$ & $42.6 \pm 6.4$ & 0.23 & 0.35 & 0.17 \\
\hline
\end{tabular}

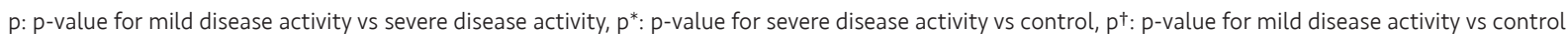
M/F: Male/Female, BMI: Body mass index, SBP: Systolic blood pressure, DBP: Diastolic blood pressure 
(6-8). Subclinical myocardial involvement cannot be shown by standard conventional echocardiography because of its limitations, such as being angle and geometry dependent and also being affected by volume load. Strain echocardiography provides segmental myocardial wall functions and is not dependent on the angle or the user.

Hensel et al. (6) showed that the global strain rate was depressed in paediatric IBD patients who were in active or remission periods in comparison to control patients. We demonstrated that children with severe disease activity at diagnosis had worse global longitudinal strain than those children with mild disease activity at diagnosis.

It was revealed that the disease activity index of CD was inversely correlated to the global longitudinal strain of LV in adult IBD patients (7). In adult patients with $C D$ and UC disease, the activity index was correlated inversely to the

Table II. Conventional and strain echocardiographic features of IBD and control patients

Demographic features of study patients

\begin{tabular}{|c|c|c|c|}
\hline & IBD & Control & $\mathrm{p}$-value \\
\hline \multicolumn{4}{|c|}{ M-Mod echocardiography } \\
\hline $\mathrm{IVSd} / \mathrm{m}^{2}(\mathrm{~mm})$ & $6 \pm 1.33$ & $5.8 \pm 1.32$ & 0.82 \\
\hline $\mathrm{LVDd} / \mathrm{m}^{2}(\mathrm{~mm})$ & $35.4 \pm 7.24$ & $35.6 \pm 8.1$ & 0.80 \\
\hline $\mathrm{LVDs} / \mathrm{m}^{2}(\mathrm{~mm})$ & $22.9 \pm 5.1$ & $22.2 \pm 4$ & 0.70 \\
\hline LVPwd/m²(mm) & $42.8 \pm 0.73$ & $43.4 \pm 0.86$ & 0.47 \\
\hline SF (\%) & $34.8 \pm 3.72$ & $37.4 \pm 5.8$ & 0.13 \\
\hline $\mathrm{EF}(\%)$ & $64 \pm 4.26$ & $67.4 \pm 5.74$ & 0.07 \\
\hline
\end{tabular}

\section{Mitral inflow parameters}

\begin{tabular}{|l|l|l|l|}
\hline Mitral E (cm/s) & $90.6 \pm 14.4$ & $98.4 \pm 16.3$ & 0.05 \\
\hline Mitral A (cm/s) & $63.3 \pm 15.8$ & $59.4 \pm 12.4$ & 0.22 \\
\hline DT (s) & $149 \pm 0.32$ & $177 \pm 0.37$ & 0.04 \\
\hline IVRT (s) & $64.7 \pm 15.5$ & $61.9 \pm 6.8$ & 0.26 \\
\hline
\end{tabular}

Strain echocardiography measurements

\begin{tabular}{|l|l|l|l|}
\hline LVAP4 (\%) & $-18.3 \pm 3.03$ & $-18.5 \pm 2.1$ & 0.70 \\
\hline LVAP3 (\%) & $-17.8 \pm 3.5$ & $-17.7 \pm 2.7$ & 0.97 \\
\hline LVAP2 (\%) & $-17.7 \pm 3.07$ & $-18.1 \pm 2.8$ & 0.54 \\
\hline GLLV (\%) & $-17.9 \pm 2.7$ & $-18 \pm 2.04$ & 0.50 \\
\hline SAXB (\%) & $-18.1 \pm 4.4$ & $-20.7 \pm 5.1$ & 0.90 \\
\hline SAXM (\%) & $-20.7 \pm 5.2$ & $-23.4 \pm 5.3$ & 0.11 \\
\hline SAXA (\%) & $-24.7 \pm 8$ & $-25.6 \pm 6.1$ & 0.69 \\
\hline GLSAX (\%) & $-23.4 \pm 9.1$ & $-23.2 \pm 3.04$ & 0.95 \\
\hline
\end{tabular}

IBD: Inflammatory bowel disease, $\mathrm{m}^{2}$ : body surface area, IVSd: Interventricular septal defect, LVDd: Left ventricular diastolic diameter, LVDs: Left ventricular systolic diameter, SF (\%): Shortening fraction, EF (\%): Ejection fraction, DT: Desceleration time, IVRT: Isovolumetric relaxation time global longitudinal strain $(7,8)$. Hensel et al. (6) found no difference in terms of myocardial functions shown by strain echocardiography between CD and UC patients. However, we showed that the longitudinal strain parameters were depressed in CD in comparison to both the control patients and the UC patients. On the other hand, we found that disease activity at diagnosis was inversely correlated to the $\mathrm{EF} \%$ and global strain value of the left ventricle in paediatric UC patients.

Chronic inflammation is associated with increased collagen type I, III, and V in IBD patients (19-23). The increased synthesis of procollagen type 3 also plays a role in the fibrosis process of other organs such as the pancreas, lungs and liver in IBD (23-25). Abnormal collagen deposition (increased serum procollagen 3 peptides) in peripheral circulation and the degradation of type I collagen was shown in IBD cases $(19,20)$. Increased procollagen type 3 is a marker used in the follow-up and prediction of the prognosis in dilated cardiomyopathy and heart failure $(26,27)$. In the adult group, the risk of cardiac diseaserelated hospitalization was found to be $37 \%$, especially in the acute exacerbation phase of the disease (28). Acute hyperinflammation is another important risk factor for myocardial decompensation. Fibrosis and fibrosis related abnormal collagen composition may be the cause of ventricular volume increases and myocardial dysfunction. Thickening of the mitral valve and mitral valve prolapse may be associated with this finding in IBD patients (29).

Altered endothelial dysfunction was shown in the gut of IBD patients (30). Endothelial dysfunction is not restricted to the gut endothelium, but it is seen in the entire endothelium as a marker of the systemic extent of the inflammation $(31,32)$.

Decreased mitral E and DT shows impairment in the early filling of the left ventricle, especially in those patients with severe disease activity. The effect of both chronic inflammation and flares of acute exacerbations on myocardial function and cardiovascular health has been shown in studies in adult IBD patients. It has been shown that adult IBD patients have coronary microvascular dysfunction leading to myocardial systolic and diastolic dysfunction (33). A study conducted with the adult population revealed that for myocardial infarction, having IBD is an independent risk factor (34).

We demonstrated the effect of disease severity at the time of diagnosis on long-term myocardial function. In paediatric patients, the negative effects of systemic inflammation on myocardium at the time of diagnosis 
Table III. Echocardiographic features and strain echocardiographic measurements in IBD patients with mild disease activity, IBD patients with severe disease activity and control patients

\begin{tabular}{|c|c|c|c|c|c|c|}
\hline & Mild disease activity & Severe disease activity & Control & p-value & $\mathbf{p}^{*}$ & $\mathrm{p}^{\dagger}$ \\
\hline IVSd/m² & $5.7 \pm 1.43$ & $6.3 \pm 1.21$ & $5.8 \pm 1.32$ & 0.64 & 0.65 & 0.39 \\
\hline $\mathrm{LVDd} / \mathrm{m}^{2}$ & $34.4 \pm 7.1$ & $37.8 \pm 4.8$ & $35.6 \pm 8.1$ & 0.43 & 0.23 & 0.43 \\
\hline LVSd/m² & $22.5 \pm 3.1$ & $23.3 \pm 4.4$ & $22.2 \pm 4.1$ & 0.56 & 0.85 & 0.21 \\
\hline LVPwd/m² & $4.4 \pm 0.61$ & $4.11 \pm 0.64$ & $4.34 \pm 0.86$ & 0.22 & 0.47 & 0.19 \\
\hline $\mathrm{SF} \%$ & $35.6 \pm 2.72$ & $33.5 \pm 3.9$ & $37.4 \pm 5.8$ & 0.36 & 0.09 & 0.09 \\
\hline $\mathrm{EF} \%$ & $66 \pm 3.16$ & $62 \pm 3.41$ & $67.4 \pm 5.74$ & 0.22 & 0.07 & 0.78 \\
\hline Mitral E & $93.7 \pm 12.4$ & $89.4 \pm 14.5$ & $98.4 \pm 16.3$ & 0.54 & 0.89 & 0.79 \\
\hline Mitral A & $64.2 \pm 14.5$ & $61.5 \pm 13.2$ & $59.4 \pm 12.4$ & 0.65 & 0.63 & 0.53 \\
\hline DT & $167 \pm 5.2$ & $131 \pm 4.9$ & $177 \pm 3.7$ & 0.05 & 0.006 & 0.46 \\
\hline IVRT & $63.7 \pm 14.5$ & $65.3 \pm 13.3$ & $61.9 \pm 6.8$ & 0.77 & 0.44 & 0.63 \\
\hline LVAP4 & $-19 \pm 2.5$ & $-17.8 \pm 3.5$ & $-18.5 \pm 2.1$ & 0.028 & 0.028 & 0.43 \\
\hline LVAP3 & $-19 \pm 2.8$ & $-16.4 \pm 3.24$ & $-17.7 \pm 2.7$ & 0.032 & 0.018 & 0.76 \\
\hline LVAP2 & $-18.2 \pm 1.98$ & $-17.4 \pm 3.5$ & $-18.1 \pm 2.8$ & 0,65 & 0.08 & 0.91 \\
\hline GLLV & $-18.7 \pm 1.79$ & $-17.1 \pm 2.9$ & $-18 \pm 2.04$ & 0,043 & 0.044 & 0.54 \\
\hline SAXB & $-18.3 \pm 4.1$ & $-18.6 \pm 3.1$ & $-20.7 \pm 5.1$ & 0.55 & 0.67 & 0.58 \\
\hline SAXM & $-20.6 \pm 5.1$ & $-21 \pm 5.9$ & $-23.4 \pm 5.3$ & 0.63 & 0.71 & 0.51 \\
\hline SAXA & $-25.6 \pm 6.5$ & $-23.6 \pm 9$ & $-25.6 \pm 6.1$ & 0.39 & 0.61 & 0.48 \\
\hline GLSAX & $-21.7 \pm 3.9$ & $-25.1 \pm 1.24$ & $-23.2 \pm 3.04$ & 0.83 & 0.72 & 0.63 \\
\hline
\end{tabular}

LVAP: Left ventricle apical chamber, GLLV: Global longitudinal left ventricle strain, SAXB: Short axis basal strain (mitral valve plane), SAXM: Short axis medial strain (papillary muscle plane), SAXA: Short axis apical strain, GLSAX: Global short axis strain, IBD: Inflammatory bowel disease

$\mathrm{p}$ : P-value for mild disease activity vs severe disease activity

$\mathrm{P}^{*}$ : P-value for severe disease activity vs control

pt: P-value for mild disease activity vs control

Table IV. Strain echocardiographic measurements of ulcerative colitis, Crohn's disease and control patients

\begin{tabular}{|c|c|c|c|c|c|c|}
\hline & UC & $C D$ & Control & p-value & $\mathbf{p}^{*}$ & $\mathrm{p}^{\dagger}$ \\
\hline \multicolumn{7}{|c|}{ M-mod echocardiography } \\
\hline $\mathrm{IVSd} / \mathrm{m}^{2}$ & $6.5 \pm 1.38$ & $6.1 \pm 1.1$ & $5.9 \pm 1.3$ & 0.12 & 0.09 & 0.08 \\
\hline $\mathrm{LVDd} / \mathrm{m}^{2}$ & $38 \pm 7.4$ & $37.4 \pm 7.7$ & $35 \pm 8.1$ & 0.45 & 0.089 & 0.067 \\
\hline $\mathrm{LVSd} / \mathrm{m}^{2}$ & $25.1 \pm 4.4$ & $23.3 \pm 3.9$ & $22.2 \pm 4$ & 0.54 & 0.61 & 0.86 \\
\hline LVPwd/m² & $4.6 \pm 0.7$ & $4.4 \pm 0.3$ & $4.3 \pm 0.8$ & 0.61 & 0.77 & 0.43 \\
\hline $\mathrm{SF}(\%)$ & $34.9 \pm 3.5$ & $36 \pm 3.7$ & $37.4 \pm 5$ & 0.21 & 0.32 & 0.27 \\
\hline $\mathrm{EF}(\%)$ & $64.5 \pm 4.5$ & $64 \pm 3.9$ & $67.4 \pm 5.7$ & 0.27 & 0.33 & 0.41 \\
\hline \multicolumn{7}{|c|}{ Mitral inflow parameters } \\
\hline Mitral E & $92.8 \pm 15.9$ & $86.7 \pm 18.5$ & $97.4 \pm 14.3$ & 0.08 & 0.038 & 0.62 \\
\hline Mitral A & $64 \pm 17$ & $58.5 \pm 17$ & $59.4 \pm 12$ & 0.21 & 0.66 & 0.12 \\
\hline DT & $142 \pm 34$ & $130 \pm 49$ & $149 \pm 28$ & 0.09 & 0.63 & 0.02 \\
\hline IVRT & $60 \pm 11$ & $69 \pm 18$ & $62 \pm 18$ & 0.15 & 0.09 & 0.28 \\
\hline \multicolumn{7}{|c|}{ Strain echocardiography measurements } \\
\hline LVAP4 & $-19.1 \pm 2.3$ & $-17.1 \pm 3.3$ & $-19 \pm 1.9$ & 0.046 & 0.048 & 0.65 \\
\hline
\end{tabular}


Polat et al.

Myocardial Functions in Inflammatory Bowel Disease

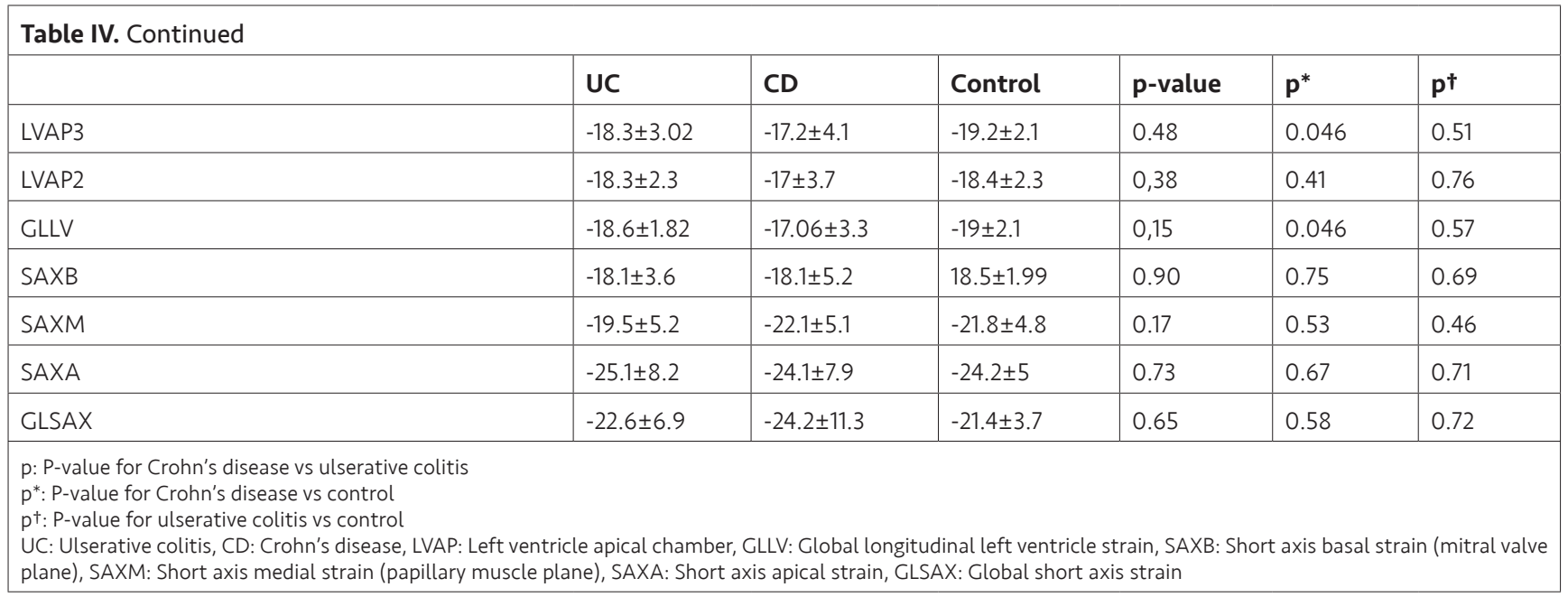

continue even after the patient is in remission. In our study, the myocardial functions of children with severe disease activity at the time of diagnosis, ascertained via strain echocardiography, were found to be impaired compared to those with mild disease activity at the time of diagnosis. We could not find any difference between the IBD and control patients in terms of myocardial functions via strain echocardiography. This may be due to the shortness of the follow-up period and the fact that the patients were in remission. We can conclude that "the more severe the inflammation is at the time of diagnosis, the more subclinical myocardial dysfunction there is in the long term".

\section{Conclusion}

Our study revealed that the severity of disease activity at the time of diagnosis has long-lasting effects on myocardial functions in those children with IBD. In this study, the systolic dysfunction of our patients was assessed after a rather short follow-up period. A longer term follow-up study to observe myocardial functions in IBD patients with both mild and severe disease activity is needed in future studies.

\section{Ethics}

Ethics Committee Approval: This study was approved by the local ethics committee in University of Health Sciences Turkey, Kanuni Sultan Süleyman Training and Research Hospital (approval number: 06, date: 29.06.2018).

Informed Consent: Written consents for the study were taken from the parents of the all patients.

Peer-review: Externally peer-reviewed.

\section{Authorship Contributions}

Design: E.P., E.E., N.G., H.A.C., Data Collection or Processing: N.G., H.A.C., Analysis or Interpretation: E.E., Literature Search: E.E., Writing: E.P., E.E.

Conflict of Interest: The authors declare that they have no conflict of interest.

Financial Disclosure: The authors declared that this study received no financial support.

\section{References}

1. Levine JS, Burakoff R. Extraintestinal manifestations of inflammatory bowel disease. Gastroenterol Hepatol (N Y) 2011; 7:235-41.

2. Harbord M, Annese V, Vavricka SR, et al. The First European Evidence-based Consensus on Extra-intestinal Manifestations in Inflammatory Bowel Disease. J Crohns Colitis 2016; 10:239-54.

3. Filimon $A M$, Negreanu L, Doca M, Ciobanu A, Preda CM, Vinereanu D. Cardiovascular involvement in inflammatory bowel disease: Dangerous liaisons. World I Gastroenterol 2015; 21:9688-92.

4. Gaduputi V, Tariq H, Kanneganti K. Abdominal aortitis associated with Crohn disease. Can J Gastroenterol Hepatol 2014; 28:69-70.

5. Andrade AR, Barros LL, Azevedo MFC, et al. Risk of thrombosis and mortality in inflammatory bowel disease. Clin Transl Gastroenterol 2018; 9:142.

6. Hensel KO, Abellan Schneyder FE, Wilke L, Heusch A, Wirth S, Jenke AC. Speckle Tracking Stress Echocardiography Uncovers Early Subclinical Cardiac Involvement in Pediatric Patients with Inflammatory Bowel Diseases. Sci Rep 2017; 7:2966.

7. Kivrak T, Sunbul $M$, Cincin A, et al. Two-dimensional speckle tracking echocardiography is useful in early detection of left ventricular impairment in patients with Crohn's disease. Eur Rev Med Pharmacol Sci 2016; 20:3249-54.

8. Cincin A, Sunbul M, Kivrak T, et al. Evaluation of cardiac function by two-dimensional speckle tracking echocardiography in ulcerative colitis patients. Dig Dis Sci 2014; 59:3004-11. 
9. Levine A, Koletzko S, Turner D, et al. ESPGHAN revised porto criteria for the diagnosis of inflammatory bowel disease in children and adolescents. I Pediatr Gastroenterol Nutr 2014; 58:795-806.

10. Levine A, Griffiths A, Markowitz I, et al. Pediatric modification of the Montreal classification for inflammatory bowel disease: the Paris classification. Inflamm Bowel Dis 2011; 17:1314-21.

11. Dotson JL, Crandall WV, Zhang P, et al. Feasibility and validity of the pediatric ulcerative colitis activity index in routine clinical practice. J Pediatr Gastroenterol Nutr 2015; 60:200-4.

12. Turner $D$, Levine $A$, Walters TD, et al. Which PCDAI Version Best Reflects Intestinal Inflammation in Pediatric Crohn Disease? I Pediatr Gastroenterol Nutr 2017; 64:254-60.

13. Hensel KO, Wilke L, Heusch A. Transthoracic Speckle Tracking Echocardiography for the Quantitative Assessment of Left Ventricular Myocardial Deformation. J Vis Exp 2016; 54736.

14. Aarestrup I, Jess T, Kobylecki C), Nordestgaard BG, Allin KH. Cardiovascular Risk Profile Among Patients With Inflammatory Bowel Disease: A Population-based Study of More Than 100 000 Individuals. J Crohns Colitis 2019; 13:319-23.

15. Freeman H), Salh B. Recurrent myopericarditis with extensive ulcerative colitis. Can / Cardiol 2010; 26:549-50.

16. Uchida W, Mutsuga M, Ito $\mathrm{H}$, Oshima $\mathrm{H}$, Usui A. Nonbacterial Thrombotic Endocarditis Associated With Crohn Disease. Ann Thorac Surg 2018; 105:199-201.

17. Efe $\mathrm{TH}$, Cimen $\mathrm{T}$, Ertem AG, et al. Atrial Electromechanical Properties in Inflammatory Bowel Disease. Echocardiography 2016; 33:1309-16.

18. Curione $M$, Barbato $M$, Amato $S$, et al. Atrioventricular block associated with Crohn's relapsing colitis in a 12-year-old child. Inflamm Bowel Dis 2010; 16:373-4.

19. Stallmach A, Schuppan D, Riese HH, Matthes $H$, Riecken EO. Increased collagen type III synthesis by fibroblasts isolated from strictures of patients with Crohn's disease. Gastroenterology 1992; 102:1920-9.

20. Kjeldsen J, Schaffalitzky de Muckadell OB, Junker P. Seromarkers of collagen I and III metabolism in active Crohn's disease. Relation to disease activity and response to therapy. Gut 1995; 37:805-10.

21. Kjeldsen J, Rasmussen M, Schaffalitzky de Muckadell OB, Kronborg O, Junker P. Collagen metabolites in the peripheral and splanchnic circulation of patients with Crohn disease. Scand I Gastroenterol 2001; 36:1193-7.

22. De Simone M, Cioffi U, Contessini-Avesani E, et al. Elevated serum procollagen type III peptide in splanchnic and peripheral circulation of patients with inflammatory bowel disease submitted to surgery. BMC Gastroenterol 2004; 4:29.

23. Leidenius MH, Risteli LT, Risteli JP, Taskinen El, Kellokumpu IH, Höckerstedt KA. Serum aminoterminal propeptide of type III procollagen (S-PIIINP) and hepatobiliary dysfunction in patients with ulcerative colitis. Scand J Clin Lab Invest 1997; 57:297-305.

24. Storch I, Sachar D, Katz S. Pulmonary manifestations of inflammatory bowel disease. Inflamm Bowel Dis 2003; 9:10415.

25. Ramos LR, Sachar DB, DiMaio C), Colombel JF, Torres J. Inflammatory Bowel Disease and Pancreatitis: A Review. I Crohns Colitis 2016; 10:95-104.

26. Kaufman BD, Videon $N$, Zhang $X$, Harris MA, Shaddy RE, Goldmuntz E. Procollagen type III amino-terminal propeptide: a serum biomarker of left ventricular remodelling in paediatric dilated cardiomyopathy. Cardiol Young 2015; 25:228-36.

27. Dupuy AM, Kuster N, Curinier C, et al. Exploring collagen remodeling and regulation as prognosis biomarkers in stable heart failure. Clin Chim Acta 2019; 490:167-71.

28. Kristensen SL, Ahlehoff O, Lindhardsen I, et al. Inflammatory bowel disease is associated with an increased risk of hospitalization for heart failure: a Danish Nationwide Cohort study. Circ Heart Fail 2014; 7:717-22.

29. Vizzardi E, Sciatti E, Bonadei I, et al. Subclinical cardiac involvement in Crohn's disease and ulcerative colitis: an echocardiographic case-control study. Panminerva Med 2016; 58:115-20.

30. Hatoum OA, Binion DG, Otterson MF, Gutterman DD. Acquired microvascular dysfunction in inflammatory bowel disease: Loss of nitric oxide-mediated vasodilation. Gastroenterology 2003; 125:58-69.

31. Roifman I, Sun YC, Fedwick JP, et al. Evidence of endothelial dysfunction in patients with inflammatory bowel disease. Clin Gastroenterol Hepatol 2009; 7:175-82.

32. WindermanR, RabinowitzSS, VaidyK, SchwarzSM. Measurement of Microvascular Function in Pediatric Inflammatory Bowel Disease. J Pediatr Gastroenterol Nutr 2019; 68:662-8.

33. Caliskan Z, Keles N, Gokturk HS, et al. Is activation in inflammatory bowel diseases associated with further impairment of coronary microcirculation? Int / Cardiol 2016; 223:176-81.

34. Aniwan S, Pardi DS, Tremaine W), Loftus EV Jr. Increased Risk of Acute Myocardial Infarction and Heart Failure in Patients With Inflammatory Bowel Diseases. Clin Gastroenterol Hepatol 2018; 16:1607-15. 